\title{
Symbiot: Congestion-driven Multi-resource Fairness for Multi-User Sensor Networks
}

\author{
Yad Tahir*, Shusen Yang ${ }^{\dagger}$, Usman Adeel*, Julie McCann* \\ ${ }^{*}$ Imperial College London, ${ }^{\dagger}$ University of Liverpool \\ \{yst11, u.adeel09, j.mccann\}@imperial.ac.uk, shusen.yang@liverpool.ac.uk
}

\begin{abstract}
In this paper, we study the problem of multi-resource fairness in multi-user sensor networks with heterogeneous and time-varying resources. Particularly we focus on data gathering applications run on Wireless Sensor Networks (WSNs) or Internet of Things (IoTs) in which users require to run a serious of sensing tasks with various resource requirements. By exploiting graph theory, queueing theory and the notion of dominant resource shares, we develop Symbiot, a light-weight, distributed algorithm that ensures multi-resource fairness between these users. With Symbiot, nodes can independently schedule its resources while maintaining network-level resource fairness through observing traffic congestion levels. Large-scale simulations based Contiki OS and Cooja network emulator show the effectiveness of Symbiot in utilizing resources and reducing average completion times.
\end{abstract}

\section{INTRODUCTION}

Wireless Sensor Networks (WSNs) and Internet of Things (IoTs) [1] are evolving towards interconnected, sensing and processing infrastructures that are expected to provide services for multiple concurrent users. One of the main reasons of having such shared enterprise deployments is to maximize the return on investment while minimizing operating costs.

Such multi-user networks are almost invariably heterogeneous in terms of their hardware components, offered resource capacities as well as user demands. Various types of sensors can be attached to nodes. The assorted capacities of some resource types, such as bandwidth, can be highly dynamic and time-varying [2], [3]. Diversity is also found when users demand specific resources. For instance, a user may require the execution of a memory-heavy task on nodes with temperature sensors, while another user may need to compute a bandwidthhungry task on nodes having humidity and light sensors.

Resource sharing in any multi-user sensor network is a key issue for one primary reason. The rapid growth of traffic and computation requirements are often not matching the growth and expansion of overall network capacity. Hence, the limited network resources should be distributed fairly between users [4], [5]. Accounting for diversity across node resources and user requirements presents an increased challenge to schedulers for fair provisioning of network resources. Getting this right is fundamental to next-generation WSN and IoT systems.

Both resource and demand heterogeneity have an impact on the efficient and fair allocation of resources to users. Current max-min-based resource allocation schemes such as [6] do not deal well with both heterogeneous resources and user demands in multi-resource systems. Utilizing multi-resource fairness

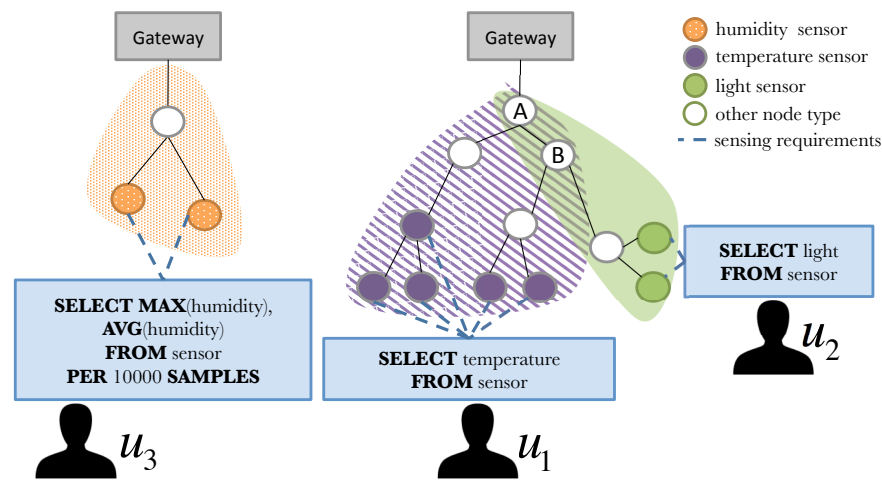

Fig. 1. A WSN with two gateways and three users. $u_{1}$ and $u_{2}$ are interested in nodes with temperature and light sensors, respectively. $u_{3}$ needs to execute a query with higher computation complexity on nodes with humidity sensors. schemes [4], [5] such as Dominant Resource Fairness (DR$F$ ) [4] have become a hot topic in both computation economics communities and cloud computing. It is proven that given users with heterogeneous demands for different resource types, these approaches achieve more efficient resource allocation than single-resource fairness schemes.

However, these approaches are limited when it comes to providing distributed scheduling that can adapt to time-varying resources. They assume that the system has a single, centralized scheduler that is aware about the resource capacities of all the system components. This means in a network with dynamic resources, nodes have to repeatedly report their available resource capacities to the scheduler. This is neither scalable nor resilient, thus impractical for large-scale networks. Furthermore, these approaches do not address multi-hop nature on which the network relies. When a user executes a task on a particular node, the output has to be forwarded to a gateway device using multi-hop communications. This creates indirect resource consumptions from the user. To ensure having fair multi-resource scheduling, it is crucial to consider both direct and indirect resource demands in multi-hop networks.

The key issue we address in this paper concerns how the available network resources, including those that are timevarying, should be allocated between competing users given the fact that each user has heterogeneous resource requirements. In particular, we consider the problem of fair resource allocation for data gathering applications in WSNs and IoTs.

To understand the complexity of the problem, consider a typical sensing application shown in Fig. 1. The network has three users $u_{1}, u_{2}$, and $u_{3}$ with different sensing require- 
ments expressed as SQL-like queries for convenience. Two cases should be analyzed here: first, based on the sensing requirements, $u_{1}$ and $u_{2}$ do not compete on resources. Each of them requires a different set of nodes. However given the fact that multi-hop communications are required to rely data, it is clear that $u_{1}$ and $u_{2}$ are now indirectly related to each other, and they compete for the shared resources of relay nodes (nodes A and B). The second case is that if $u_{1}$ changed its requirements and asked for all the resources available in all nodes with temperature or light sensors, surely this would be considered unfair according to the max-min fairness model as $u_{1}$ would consume considerably more network resources than $u_{2}$. From these two cases there are two key questions that have to be carefully addressed: First, how can we formally define the relationship between users? $u_{1}$ and $u_{2}$ are dependent in terms of resource requirements, but $u_{3}$ certainly is not. Second, given each user submit sensing tasks to the network, what is the maximum number of tasks that each user can execute while the resource consumptions between related users remain fair?

We present the study of the fair resource allocation problem in network with multiple, time-varying resource types. The contributions of this paper are summarized as follows:

1. We establish a set of system models to formally describe the components of multi-user WSNs and IoT. By exploiting graph theory, we formally define the relationship between users in terms of their resource requirements. (Section II)

2. We propose a new extension to max-min fairness that exploits graph theory and dominant resource sharing to ensure multi-resource fairness between users running a series of sensing tasks. We develop a new algorithm, named Symbiot, that uses iterative linear programming and queueing theory to compute the proposed fairness extension. Symbiot is not only lightweight, but also it is fully distributed eliminating the need for centralized scheduling. Through observing local resource capacities and current traffic congestion levels, nodes can independently allocate resources for its users while maintaining multi-resource fairness between related users in the whole network. To the best of our knowledge, this work is not only the first work to address the indirect requirement problem and utilize queueing theory in multi-resource scheduling, but also there has been no prior work that has proposed a distributed solution to achieve multi-resource fairness. (Section III)

3. We implemented Symbiot on Contiki OS [7], an open source operating system for wireless sensor networks and IoT. We integrated Symbiot with the IPv6 stack provided by Contiki. Through large-scale simulations with the Cooja network emulator [7], we demonstrate the practical performance of Symbiot algorithm. Our experiments show that Symbiot outperforms the naive version of distributed DRF in terms of reducing the average of job completion times. (Section IV)

\section{SySTEM Models}

We consider a WSN or IoT that consists of a set of wireless nodes $\mathcal{N}=\mathcal{S} \cup \mathcal{H}$ communicating in a multi-hop fashion, where $\mathcal{S}$ represents the set of all nodes that can sense, generate and relay data packets; while $\mathcal{H}$ is the set of all gateways that collect the data traffic produced by the network. Let the set $\mathcal{N}_{x}$ holds all one-hop neighbors that node $x \in \mathcal{N}$ that can communicate with. The network has a set of users $\mathcal{U}$ and we assume that the network operates in discrete time with a unit time slot (e.g. a second) $t \in\{1,2, \ldots\}$.

\section{A. Node Operations and Data Queues}

Node $n \in \mathcal{S}$ can run two types of tasks: sensing and data forwarding.

Each user $u \in \mathcal{U}$ can execute sensing tasks periodically on nodes. We assume that a sensing task requires no more than one time slot to finish. We assume that the sensing tasks are delay-tolerant, which is realistic as many environmental monitoring applications are reasonably delay tolerant.

Performing sensing tasks will generate data packets to be collected by a gateway $h \in \mathcal{H}$. To achieve this, nodes execute data forwarding tasks. The data can be transmitted directly if $h \in \mathcal{N}_{n}$, or indirectly in a multi-hop fashion if $h \notin \mathcal{N}_{n}$. The underlinying routing layer determines the forwarding policy. In this paper, we only consider networks running in a fixed routing scenario, i.e., the route of each data flow is predetermined and fixed during the time of interest. We also assume both sensing and forwarding tasks are indivisible.

In the network, each node maintains data packets through queues (data buffers). Let $Q_{n}^{u}$ denote the queue existing in node $n$ to hold the data packets generated by user $u$. Let $Q_{n}^{u}(t)$ be the queue backlog (or queue length) of the queue $Q_{n}^{u}$ at time slot $t$. The queue dynamics of $Q_{n}^{u}$ are defined as follows:

$$
\begin{aligned}
& 0 \leq Q_{n}^{u}(t) \leq \operatorname{Max} Q_{n}^{u} \\
& Q_{n}^{u}(t+1)=\left|Q_{n}^{u}(t)-f_{u, n}^{\text {out }}(t)\right|_{+}+r_{u, n}(t)+f_{u, n}^{\text {in }}(t)
\end{aligned}
$$

Where the operator $|\cdot|_{+}$represents $\max (0,$.$) and \operatorname{Max} Q_{n}^{u}>$ 0 is the maximum queue length (i.e. allocated data buffer size) of $Q_{n}^{u} . r_{u, n}(t)$ is the output of performing sensing tasks in node $n$ for user $u$ at time slot $t . f_{u, n}^{i n}(t)$ and $f_{u, n}^{\text {out }}(t)$ are the incoming and outgoing traffic of node $n$ for user $u$, respectively. It is worth noting that the queue length of any gateway always equals to zero, i.e.

$$
Q_{h}^{u}(t)=0, h \in \mathcal{H}, \forall u \in \mathcal{U}, t \geq 1
$$

\section{B. Node Resources}

Each node $n \in \mathcal{N}$ offers $K$ types of resources for users such as computing (i.e. MCU), memory, bandwidth, and light sensors. Let a $K$-dimensional vector $\mathbf{c}_{n}(t)=$ $\left(c_{n, 1}(t), \ldots, c_{n, K}(t)\right)$ represents node's resource capacities at time slot $t$, where each entry $c_{n, k}(t) 1 \leq k \leq K$ represents the resource capacity of $k$ th resource of $n$ at $t$. Let $\mathbf{C}(t)$ be a $|\mathcal{N}| \times K$-dimensional matrix representing the resource capacity for the whole network at $t$.

In the rest of this paper, we will use a tuple $(n, k), n \in$ $\mathcal{N}, 1 \leq k \leq K$ to refer to a specific resource.

\section{User Resource Requirements}

Let $\widehat{\mathbf{R}}^{u}$ be a $|\mathcal{N}| \times K$-dimensional matrix in which the entry $\widehat{\mathbf{R}}_{n, k}^{u}$ represents the requirement from $(n, k)$ to perform 
a sensing task by user $u$. Let $\widetilde{\mathbf{R}}^{u}$ be a $|\mathcal{N}| \times K$-dimensional matrix in which the entry $\widetilde{\mathbf{R}}_{n, k}^{u}$ is the amount of resource needs from $(n, k)$ to perform a data forwarding task on $Q_{n}^{u}$.

The gross resource requirements of user $u$ is represented as a $|\mathcal{N}| \times K$ matrix $\mathbf{R}^{u}$, where:

$$
\mathbf{R}_{n, k}^{u}=\widehat{\mathbf{R}}_{n, k}^{u}+\widetilde{\mathbf{R}}_{n, k}^{u}
$$

Consider $\mathcal{D}_{u}$ to represent the set of strictly positive resource demands of user $u$ :

$$
\mathcal{D}_{u}:=\left\{(n, k): \mathbf{R}_{n, k}^{u}>0, n \in \mathcal{N}, 1 \leq k \leq K\right\}
$$

\section{Topological User Dependency}

This section presents a graph model to characterize the dependency of all users in $\mathcal{U}$, with respect to resource requirements.

Definition 1. [User Dependency Graph]. We can use an undirected graph $G(\mathcal{U}, \mathcal{V})$ to represent the dependency of all users, where

$$
\mathcal{V}:=\left\{(u, v): u, v \in \mathcal{U}, \mathcal{D}_{u} \cap \mathcal{D}_{v} \neq \emptyset\right\}
$$

is the set of the links in the graph. Each link $(u, v) \in \mathcal{L}$ indicates that two users $u$ and $v$ share some resources.

Definition 2. [User Dependent Cluster (UDC)]. For a given user dependence graph $G(\mathcal{U}, \mathcal{V})$, a User Dependent Cluster (UDC) $\mathcal{C} \subseteq \mathcal{U}$ is defined as the set of users in a connected component ${ }^{1}$ of $G(\mathcal{U}, \mathcal{V})$.

Define $\mathcal{U}_{n, k}$ as the set of users that have a strictly positive demand of resource $(n, k), n \in \mathcal{N}, 1 \leq k \leq K$, i.e.

$$
\mathcal{U}_{n, k}:=\left\{u: \mathbf{R}_{n, k}^{u}>0, u \in \mathcal{U}\right\}
$$

It can be seen that two users $u_{1}$ and $u_{2}$ in a UDC $\mathcal{C}$ may not share common resources, meaning that they are not directly dependent on each other. However the resource requirements of $u_{1}$ would affect that of all neighbors $v \in \mathcal{U}_{n, k},(n, k) \in$ $\mathcal{D}_{u_{1}}$. By repeating the above process, the requirement of $u_{1}$ would affect all users in $\mathcal{C}$, including $u_{2}$. This implies that all users in a UDC are directly or indirectly dependent in terms of resource requirements.

\section{E. Objective}

The objective of this paper is to develop a distributed, fair scheduling algorithm to allocate resources for sensing and data forwarding tasks for the users of the network. In particular for every time slot $t$, it is required to distributedly compute two $|\mathcal{N}| \times|\mathcal{U}|$-dimensional matrices $\widehat{\mathbf{X}}(t)$ and $\widetilde{\mathbf{X}}(t)$, where the entries $\widehat{\mathbf{X}}_{u, n}(t)$ and $\widetilde{\mathbf{X}}_{u, n}(t)$ are the number of sensing and forwarding tasks performed by user $u$ in node $n$, respectively.

The network resources allocated to user $u$ at time slot $t$ is defined as a $|\mathcal{N}| \times K$-dimensional matrix $\mathbf{A}^{u}(\widehat{\mathbf{X}}(t), \widetilde{\mathbf{X}}(t))$ where each entry $\mathbf{A}_{n, k}^{u}(t)$ represents the amount of resource $(n, k)$ allocated to user $u$ at time slot $t$ :

\footnotetext{
${ }^{1}$ In graph theory, a connected component is subgraph in which any two vertices are connected to each other by paths, and which is connected to no additional vertices in the supergraph.
}

$$
\mathbf{A}_{n, k}^{u}(t)=\widehat{\mathbf{X}}_{u, n}(t) \widehat{\mathbf{R}}_{n, k}^{u}+\widetilde{\mathbf{X}}_{u, n}(t) \widetilde{\mathbf{R}}_{n, k}^{u}
$$

Definition 3. [Feasible Resource Allocation]. A resource allocation matrix $\boldsymbol{A}^{u}(\widehat{\mathbf{X}}(t), \widetilde{\mathbf{X}}(t))$ is feasible if the following condition is satisfied:

$$
\sum_{u \in \mathcal{U}} \mathbf{A}^{u}(\widehat{\mathbf{X}}(t), \widetilde{\mathbf{X}}(t)) \preceq \mathbf{C}(t)
$$

where $\preceq$ means entry-wise smaller than or equal to.

\section{SYMBIOT}

Let $d_{u, n, k}(t)=\mathbf{R}_{n, k}^{u} / c_{n, k}(t)$ be the resource demand share of user $u$ for resource $(n, k)$ at slot $t$. Let $d_{u, n}^{\max }(t)$ denote the node-wise dominant demand share of user $u$ in node $n$.

$$
d_{u, n}^{\max }(t)=\max _{1 \leq k \leq K} d_{u, n, k}(t)
$$

We define $d_{u, k}(t)$ as the normalized resource demand share of a user $u$ for resource $(n, k)$ :

$$
d_{u, k}(t)=d_{u, n, k}(t) / d_{u, n}^{\max }(t)
$$

For each user $u$ in a UDC $\mathcal{C}$, define $U D C$-wise dominant demand share as

$$
d_{u}^{\max }(t)=\max _{(n, k) \in \mathcal{D}(\mathcal{C})} d_{u, n}^{\max }(t), u \in \mathcal{C}
$$

where

$$
\mathcal{D}(\mathcal{C}):=\bigcup_{u \in \mathcal{C}} \mathcal{D}_{u}
$$

denotes the set of all resources used by all users in $\mathcal{C}$. For a given $d_{u}^{\max }(t)$, we donate the bottleneck node $n^{*}$ as the node which provides $d_{u}^{\max }(t)$, i.e.:

$$
n^{*}=\arg \max _{n} d_{u, n, k}(t),(n, k) \in \mathcal{D}(\mathcal{C}), u \in \mathcal{C}
$$

We define the $U D C$-wise dominant share for user $u$ in $\mathcal{C}$ as:

$$
d_{u}^{\max }(t)\left(\widehat{\mathbf{X}}_{u, n^{*}}(t)+\widetilde{\mathbf{X}}_{u, n^{*}}(t)\right)
$$

\section{A. Algorithm}

The basic idea of Symbiot is to implement a distributed, light-weight algorithm to approximate the max-min fairness for the UDC-wise dominant shares for each UDC.

Because the algorithm is distributed, every node $n \in \mathcal{N}$ computes resource scheduling locally. However, we utilize network traffic congestion to collect feedback from the bottleneck nodes existing in UDCs. The pseudo code of Symbiot is summarized in Fig. 2 and Fig. 3.

In node $n$ at the beginning of time slot $t$, all users that use $n$ are considered to be unsaturated (line 01 in Fig. 2) and stored in the unsaturated user set $\dot{\mathcal{U}}_{u s}$. Symbiot allocates resources for the unsaturated users through multiple iterations (lines 02-21 in Fig. 2). In each iteration Symbiot finds a budget $b$ that maximizes the equalized node-wise dominant demand 


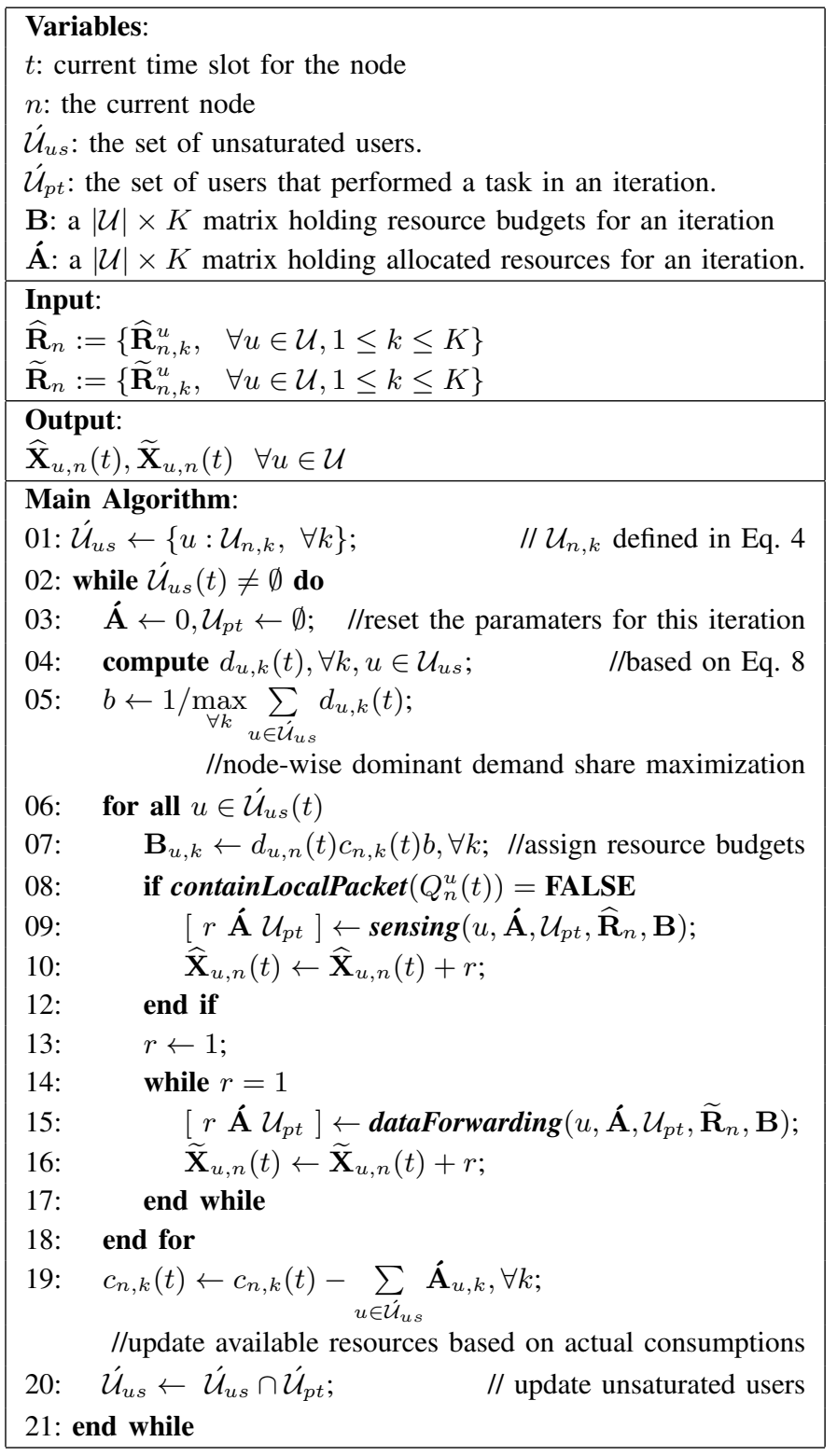

Fig. 2. Symbiot - Main Algorithm

shares of these users (lines 04-05 in Fig. 2) by computing $b=$ $1 / \sum_{u \in \dot{\mathcal{U}}_{u s}} \frac{d_{u, n, k}(t)}{d_{u, n}^{\max }(t)}$. This also ensures that Symbiot adheres to the capacity constraint introduced in the Definition 3.

Then Symbiot assigns each unsaturated user a resource budget for every resource type $k$ (line 07 in Fig. 2). The given resource budgets can be utilized for sensing tasks, data forwarding tasks or both. For sensing tasks, Symbiot first checks whether the data queue of the user contains a local packet (i.e. generated by $n$ ). If no local packet found, Symbiot executes the sensing function. It is worth noting that in the pseudo code of Symbiot, we assume the data queues of the users follow the First-In-First-Out (FIFO) scheduling. Symbiot utilizes the remaining budget for data forwarding tasks by calling the dataForwarding function. As shown in Fig. 3, both sensing and dataForwarding update the resource consumptions and active users set $\dot{\mathcal{U}}_{p t}$ for the current iteration. These functions returns one if the sensing or data $\operatorname{sensing}\left(u, \mathbf{A}, \mathcal{U}_{p t}, \widehat{\mathbf{R}}_{n}, \mathbf{B}\right)$
$01:$ if $Q_{n}^{u}(t) \neq \operatorname{Max} Q_{n}^{u} \wedge \exists k \widehat{\mathbf{R}}_{n, k}^{u} \neq 0 \wedge$

$$
\forall k \dot{\mathbf{A}}_{u, k}+\widehat{\mathbf{R}}_{n, k}^{u} \leq \mathbf{B}_{u, k}
$$

//if $Q_{n}^{u}$ is not full, user requires sensing, and sufficient budget

02: $\quad \dot{\mathbf{A}}_{u, k} \leftarrow \hat{\mathbf{A}}_{u, k}+\widehat{\mathbf{R}}_{n, k}^{u}, \forall k ; \quad$ //allocate the resources

03: executeSensingTask $(u)$; //sensing and storing data in $Q_{n}^{u}$

04: $\quad \dot{\mathcal{U}}_{p t}(t) \leftarrow \dot{\mathcal{U}}_{p t}(t) \cup\{u\}$;

05: return $\left[1 \hat{\mathbf{A}} \mathcal{U}_{p t}\right.$ ];

06: end if

07: return [ 0 Á $\mathcal{U}_{p t}$ ];

dataForwarding $\left(u, \mathbf{A}, \mathcal{U}_{p t}, \widetilde{\mathbf{R}}_{n}, \mathbf{B}\right)$

08: $p \leftarrow \operatorname{getNextHop}() ; / /$ node to where packets should be sent 09: if $Q_{n}^{u}(t) \neq 0 \wedge Q_{p}^{u}(t) \neq \operatorname{Max} Q_{p}^{u} \wedge \forall k \dot{\mathbf{A}}_{u, k}+\widetilde{\mathbf{R}}_{n, k}^{u} \leq \mathbf{B}_{u, k}$ //check $Q_{n}^{u}$ is not empty, $Q_{n}^{p}$ is not full, and sufficient budget 10: $\quad \dot{\mathbf{A}}_{u, k} \leftarrow \dot{\mathbf{A}}_{u, k}+\widetilde{\mathbf{R}}_{n, k}^{u}, \forall k ; \quad$ //allocate the resources 11: $\quad$ sendDataPacket $(u, p) ; \quad$ //sends a packet from $Q_{n}^{u}$ to $p$

12: $\quad \dot{\mathcal{U}}_{p t} \leftarrow \dot{\mathcal{U}}_{p t} \cup\{u\}$;

13: return $\left[\begin{array}{lll}1 & \mathbf{A} & \mathcal{U}_{p t}\end{array}\right]$

14: end if

15: return $\left[0\right.$ Á $\mathcal{U}_{p t}$ ];

Fig. 3. Symbiot - Sensing and Data Forwarding functions

forwarding tasks is actually executed, otherwise zero. Based on the feedback given in the lines 09 and 15 in Fig. 2, Symbiot updates the $\widehat{\mathbf{X}}_{u, n}(t)$ and $\widetilde{\mathbf{X}}_{u, n}(t)$ outputs for all unsaturated users. Then the remaining resource capacities are adjusted according to the resource consumptions of this iteration. In line 20 in Fig. 2, Symbiot removes saturated users from $\dot{\mathcal{U}}_{\text {us }}$. We define a saturated user as a user who did not perform any task, whether sensing or data forwarding, in an iteration. This process continues until all users become saturated.

Remark 1. Both sensing and data forwarding operations are traffic-aware. Even if a user has the required budget to perform a task, Symbiot will not allocate resources if traffic congestion is observed for that user. In line 01 in Fig. 3, sensing tasks check whether the queue of the current node is not full. Line 09 in Fig. 3 ensures data forwarding tasks are not performed if the queue of the next-hop node is not full.

Remark 2. In a bottleneck node, the data queues of some users will be full. A bottleneck node $n^{*}$ in a UDC will limit the budget of one or more users as they are seen to be locally unfair with respect to other users. This means in the node $n^{*}$, the $f_{u, n^{*}}^{\text {out }}<f_{u, n^{*}}^{i n}$ for some users. Based on the queue dynamics defined in Eq. 1, the queue levels of some $Q_{n^{*}}^{u}$ will gradually increase until it reaches the corresponding $\operatorname{Max} Q_{n^{*}}^{u}$.

Remark 3. Bottleneck nodes in a UDC cause traffic congestion for nodes sending data packets in. According to Remark 2, when $Q_{n^{*}}^{u}=\operatorname{Max} Q_{n^{*}}^{u}, n *$ also limits data forwarding operations for nodes that send data directly to $n^{*}$ as described in Remark 1. Eventually when their data queues become full, this results in affecting the sensing operations performed on these nodes. This process repeats itself throughout the UDC and will affect all nodes that generating and sending data packet for user $u$ through the $n^{*}$. 


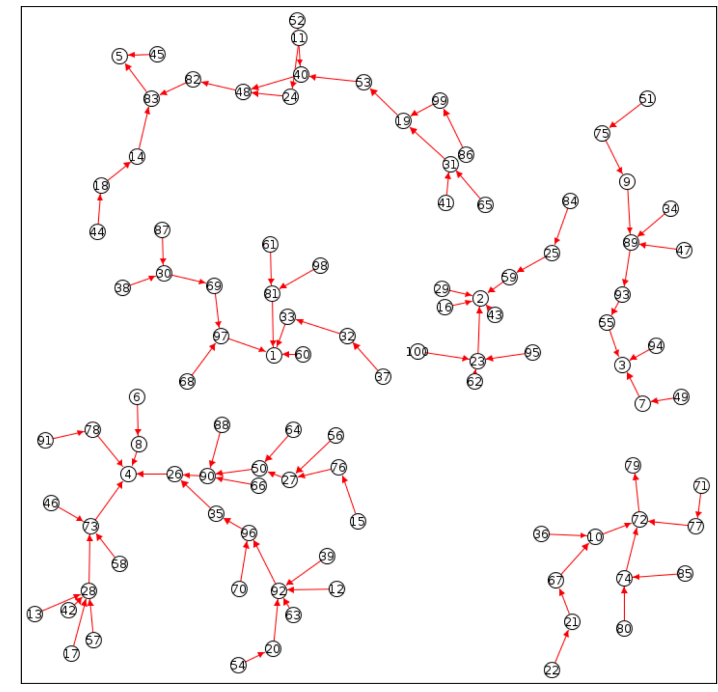

Fig. 4. The network topology of the experiments. IV. EVALUATION

The performance of Symbiot was evaluated through conducting a set of simulation on Cooja. We implemented Symbiot on top of Contiki OS [7] and its IPv6 stack. The implementation provides a set of public APIs to allow users to express their sensing and data forwarding requirements. Internally it uses UDP messages and IPv6 to transfer data packets from nodes to gateways. We use ICMPv6 to periodically broadcast the queue lengths of the users between nodes.

We established a 100-node network with 5 gateways and 95 nodes. The network topology of our experiments is shown in Fig. 4. The network uses CSMA and RPL with the default parameter settings for the MAC and routing layers, respectively. We set RPL to use the ETX Objective Function. The nodes employ IEEE 802.15.4 transceiver, CC2420. Based on the experimental studies in [8], the bandwidth capacity of the nodes is set to be 160 40-bytes packets per second. The nodes in our network uses MSP430F1611 microcontroller with 10KB RAM. The network offers two types of sensors: temperature and humidity with the resource capacities of 1000 and 2000 readings per second, respectively. We randomly chose 40 nodes to offer temperature sensing and another 40 nodes for humidity sensing. In all simulations the duration of a time slot was set as one second and $\operatorname{Max}_{n}^{u}$ for all users in all nodes were equal to 70 .

In the reset of the paper, we will use the vector $\left\langle r_{1}\right.$ packets, $r_{2}$ bytes of RAM, $r_{3}$ temperature readings, $r_{4}$ humidity readings $\rangle$ to represent the resource requirements of offered resource types in the experiments.

\section{A. Dynamic Resource Allocation}

In our first experiment, we show how Symbiot dynamically allocates resources between users in a system with timevarying resource requirements and capacities. Fig. 5 shows the average allocated network resources and UDC-wise shares for each users as a function of time.

In the beginning, the network has two users $u_{1}$ and $u_{2}$. $u_{1}$ has the resource requirements of $\langle 0,1,2,0\rangle$ for sensing and $\langle 1,20,0,0\rangle$ for data forwarding. Similarly, $u_{2}$ needs
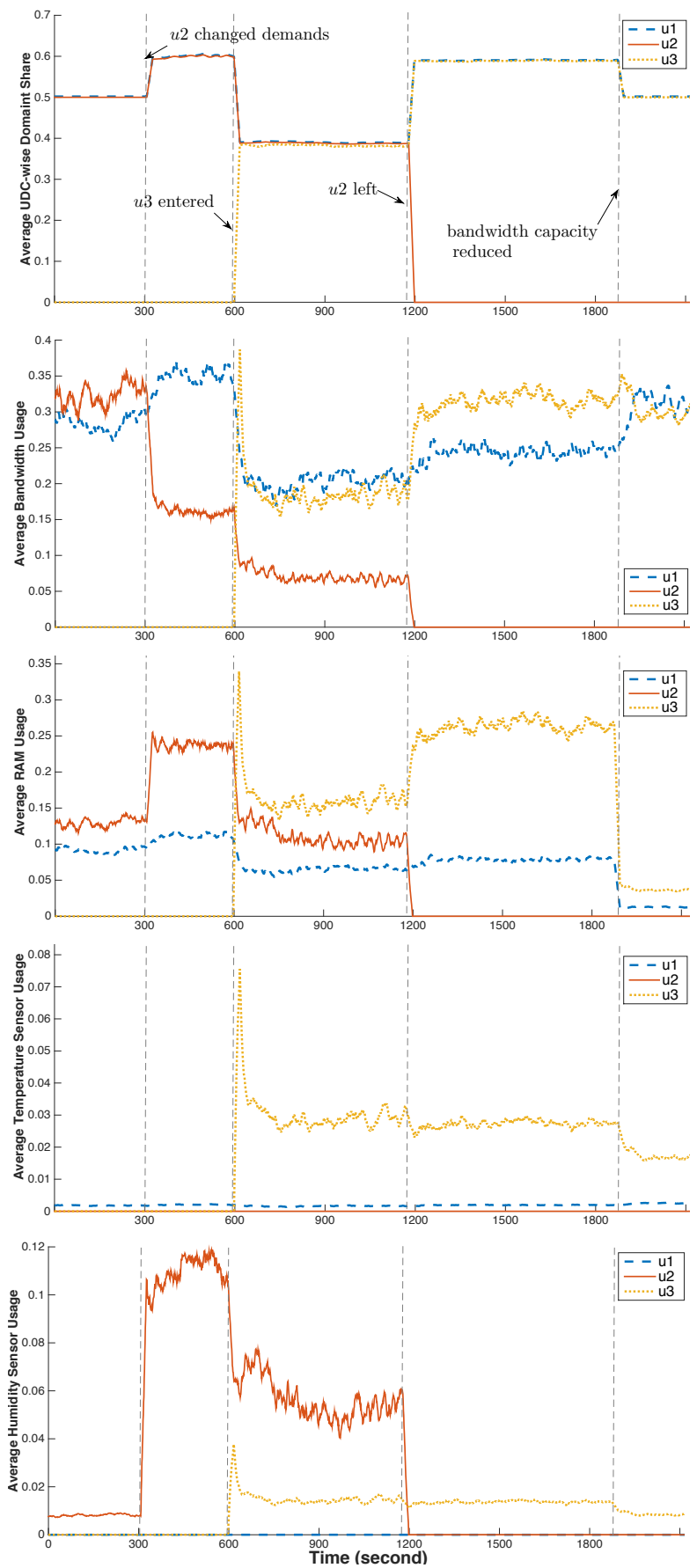

Fig. 5. Symbiot resource allocation in a dynamic system.

$\langle 0,10,0,20\rangle$ for sensing and $\langle 2,50,0,0\rangle$ for data forwarding operations. As seen in Fig. 5, the UDC-wise dominant shares for both users are equal to $50 \%$ as bandwidth is the most demanded resource for both users in bottleneck nodes. At slot $300, u_{2}$ changes his/her resource requirements making it more memory demanding. $u_{2}$ now demands $\langle 0,2048,200,0\rangle$ for sensing and $\langle 9,512,0,0\rangle$ for data forwarding. Symbiot dynamically adjusts the resource allocation for both users. The UDC-wise dominant shares increased to around $60 \%$ as the most required resource for $u_{1}$ is bandwidth while for $u_{2}$ now it becomes RAM. At time slot $600, u_{3}$ enters the 
network with the resource requirements of $\langle 0,200,20,20\rangle$ for sensing and $\langle 4,200,0,0\rangle$ for data forwarding. Symbiot lowers the resource allocation for both $u_{1}$ and $u_{2}$ to accommodate the resource demands for the new users. As $u_{3}$ enters the network, the nodes in beginning tries to perform as much sensing as possible until bottleneck nodes are found causing traffic congestion. This will result in gradually lowering the sensing rate as shown between slot 600 and 700 . At slot 900 when $u_{2}$ leaves the network, Symbiot allocates the released resources from $u_{2}$ to $u_{1}$ and $u_{3}$. At time slot 1900 , we intentionally reduce the bandwidth capacities of all nodes by $50 \%$. Symbiot automatically adjusts the allocations for both $u_{1}$ and $u_{3}$.

\section{B. Symbiot vs Alternative Multi-Resource Allocation}

To compare the performance of Symbiot with another multiresource allocation algorithm, we implemented a naive version of distributed DRF. Here, each node runs the progressive filling of DRF [4]. However, the sensing and data forwarding tasks are not traffic-aware. If any node receives data packets more than $\operatorname{Max} Q_{n}^{u}$, the node will drop the packets. In these experiments, we measured the completion times required to perform sensing tasks on all the nodes required by the users.

Fig. 6 presents the reductions of average completion times in networks with different numbers of gateways. Here, the network has two users with sensing requirements of $\langle 0,1,2,0\rangle$ and $\langle 0,10,0,20\rangle$ and forwarding requirements of $\langle 1,20,0,0\rangle$ and $\langle 2,50,0,0\rangle$. Fig. 7 shows Symbiot outperforms the implemented distributed DRF in networks with different numbers of users. The used topology had 5 gateways and the sensing and forwarding requirements were randomly generated.

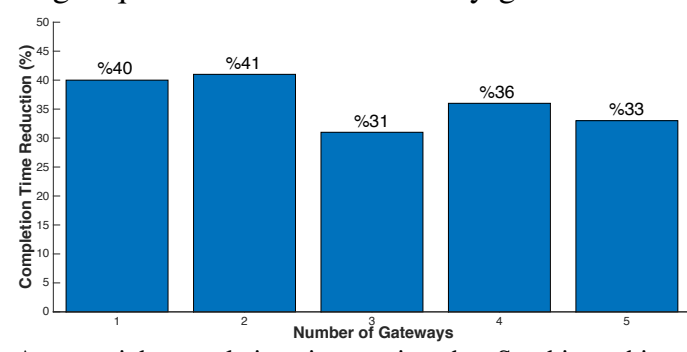

Fig. 6. Average job completion time saving that Symbiot achieved against naive, distributed DRF in networks with different numbers of gateways.

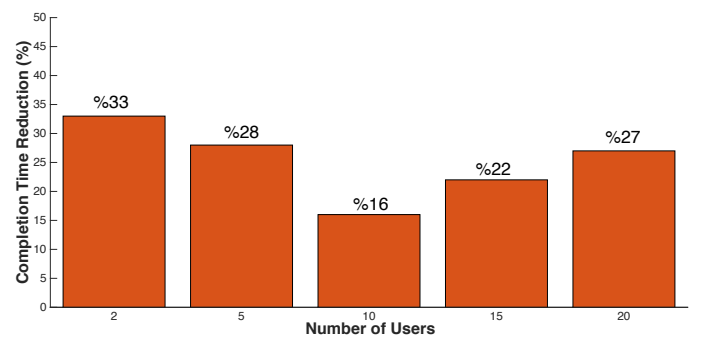

Fig. 7. Average job completion time saving that Symbiot achieved against the implemented version of distributed DRF in networks with different users.

\section{RELATED WORK}

One way to ensure fairness in multi-resource systems is to employ single-resource abstraction models where system resources are divided into fixed partitions, often referred as slots. As pointed out in [4], employing such simple abstraction models are considered to be inefficient when users have heterogeneous demands for resources. Ghodsi et al. [4] propose
Dominant Resource Fairness (DRF) as an alternative approach to ensure fairness in multi-resource systems. DRF satisfies several highly desirable fairness properties, and it quickly received significant attention from the research community. Parkes et al. [9] extend the DRF paradigm to provide a compelling solution for weighted and zero demands users. In fact, the node-wise dominant share computations in SymbIoT is greatly inspired by [9] work. Wang et al. [10] suggest to the use of the notion of global dominant shares to address the heterogeneity of server capabilities in cloud computing. All the approaches above focus on systems with centralized schedulers. As discussed in Section, deploying centralized schedulers can be very expensive and not feasible for networks with time-varying resources. We purpose a solution to distributedly allocating resources for users. We also show how indirect resource requirements coming from multi-hop communications should be handled. This is crucial for ensuring multi-resource fairness accross the whole network.

\section{CONCLUSION}

This paper addresses the problem of multi-resource fairness between users in data gathering applications in WSNs and IoT. Here, nodes can offer multiple types of resources with heterogeneous, time-varying capacities. Users can have different requirements on various resource types. We explain why the current state-of-the-art falls short when it comes to achieve multi-resource fairness in such networks. By utilizing graph theory, queueing theory and dominant resource sharing, we propose Symbiot, a lightweight, distributed algorithm as a solution to this problem. Our simulations based on Cooja network emulator shows the practical performance of Symbiot.

\section{REFERENCES}

[1] Z. Sheng, S. Yang, Y. Yu, A. Vasilakos, J. Mccann, and K. Leung, "A survey on the ietf protocol suite for the internet of things: Standards, challenges, and opportunities," IEEE Wireless Commun., vol. 20, no. 6, pp. 91-98, 2013.

[2] S. Yang, U. Adeel, and J. McCann, "Selfish mules: Social profit maximization in sparse sensornets using rationally-selfish human relays," IEEE JSAC, vol. 31, no. 6, pp. 1124-1134, 2013.

[3] S. Yang, X. Yang, J. A. McCann, T. Zhang, G. Liu, and Z. Liu, "Distributed networking in autonomic solar powered wireless sensor networks," IEEE J. Sel. Areas Commun., vol. 31, no. 12, pp. 750-761, 2013.

[4] A. Ghodsi, M. Zaharia, B. Hindman, A. Konwinski, S. Shenker, and I. Stoica, "Dominant Resource Fairness: Fair allocation of multiple resource types," in Proc. USENIX NSDI, 2011.

[5] L. Popa, G. Kumar, M. Chowdhury, A. Krishnamurthy, S. Ratnasamy, and I. Stoica, "Faircloud: Sharing the network in cloud computing," in Proc. ACM SIGCOMM, 2012.

[6] S. Yang and J. A. McCann, "Distributed optimal lexicographic max-min rate allocation in solar-powered wireless sensor networks," ACM Trans. Sensor Networks, vol. 11, no. 1, p. 9, 2014.

[7] "Contiki: The Open Source OS for the Internet of Things." [Online]. Available: http://www.contiki-os.org

[8] A. Sridharan and B. Krishnamachari, "Explicit and precise rate control for wireless sensor networks," in Proc. ACM SenSys, 2009, pp. 29-42.

[9] D. Parkes, A. Procaccia, and N. Shah, "Beyond Dominant Resource Fairness: Extensions, limitations, and indivisibilities," in Proc. ACM EC, 2012.

[10] W. Wang, B. Li, and B. Liang, "Dominant Resource Fairness in cloud computing systems with heterogeneous servers," in Proc. IEEE INFOCOM, 2014. 